\title{
MicroRNA in localized scleroderma: a review of literature
}

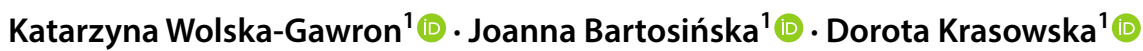

Received: 10 February 2019 / Revised: 8 September 2019 / Accepted: 3 October 2019 / Published online: 21 October 2019

(c) The Author(s) 2019

\begin{abstract}
Localized scleroderma ( $\mathrm{LoSc})$ is rare connective tissue disease that manifests with inflammation and fibrosis of the skin. Depending on the LoSc subtype, adjacent structures such as subcutaneous tissue, fascia, muscles, bones may be affected. The hallmark of fibrosis is tissue remodelling with excess deposition of extracellular matrix proteins (ECM), principally collagens. MicroRNAs (miRNAs) are small, noncoding RNA molecules that consist of 19-24 nucleotides and act as negative regulators of gene expression at the posttranscriptional level. Based on the current articles, approximately 40 microRNAs have been linked to fibrosis in different organs and diseases. The majority of these molecules promote or inhibit fibrosis by targeting connective tissue growth factor (CTGF), extracellular matrix proteins, TGF- $\beta$ pathway and MAPK (mitogenactivated protein kinase) pathway. Further, particular microRNAs regulate fibrogenesis by altering epithelial-to-mesenchymal transition (EMT) or activating proliferation of myofibroblasts. MiRNAs are relatively stable, detectable in tissues and body fluids (serum, plasma) which suggest that they may serve as beneficial biomarkers to monitor the course of the disease and response to treatment. Herein, we report the present state of knowledge on microRNA expression in localized scleroderma.
\end{abstract}

Keywords Localized scleroderma $\cdot$ Morphea $\cdot \operatorname{LoSC} \cdot$ MicroRNA $\cdot$ miRNA

\section{Localized scleroderma}

Localized scleroderma is a rare, autoimmune disease that affects dermis and sometimes structures lying beneath the skin. The incidence rate is reported to range from 0.4 to 2.7 cases per 100000 people [14]. Females are more frequently affected then men (2.6-6 times) [15]. According to German classification, localized scleroderma may be divided into five clinical subtypes-limited, generalized, linear, deep and mixed $[14,15]$. Circumscribed morphea is the most common type that usually affects adults between 40 and 50 years of age $[15,20]$. Linear LoSc is frequently present in children aged 2-14 [15]. The disease outcome relies on the subtype of localized scleroderma and extent of skin lesions. Delayed diagnosis may lead to time lapse in therapy and, in consequence, functional disabilities and disfiguration $[14,15]$. The etiopathogenesis of localized scleroderma has not been fully understood yet, however, a number of immunoinflammatory

Katarzyna Wolska-Gawron

wolskak@poczta.onet.pl

1 Chair and Department of Dermatology, Venerology and Paediatric Dermatology, Medical University of Lublin, Lublin, Poland and profibrotic factors are likely implicated (Fig. 1) [3, 26, 30]. Evidence points to LoSc as an autoimmune disorder due to family/personal history of autoimmune diseases, presence of certain human leucocyte antigen (HLA) subtypes and high prevalence of autoantibodies. $10-30 \%$ of patients with localized scleroderma reported having a family history of autoimmune disease, whereas $10 \%$ of them present with a concomitant autoimmune disorder [especially vitiligo, alopecia areata, rheumatoid arthritis (RA)] [30]. Studies examining HLA association with LoSc revealed that HLA-B*37 and HLA-DRB $1 * 04: 04$ lead to increased susceptibility, especially in linear and generalized subtypes of LoSc [26, 30]. Additionally, HLA-DRB $1 * 04: 04$ strongly correlates with increased risk for rheumatoid arthritis what implies a common genetic susceptibility to LoSc and RA [30]. Cases of linear localized scleroderma, manifesting with skin lesions following Blaschko's lines, confirm a potential relevance of mosaicism [26, 30]. Epigenetic mechanisms may stand for a feasible link between genetic and external (environmental) factors [3, 26]. Over the last decade, microRNAs have attracted tremendous interest as a pivotal epigenetic regulators in LoSC. Additionally, deregulated histone acetylation and DNA methylation have been demonstrated in localized scleroderma [26]. 
Fig. 1 The etiopathogenesis of localized scleroderma $[3,26$, 30]
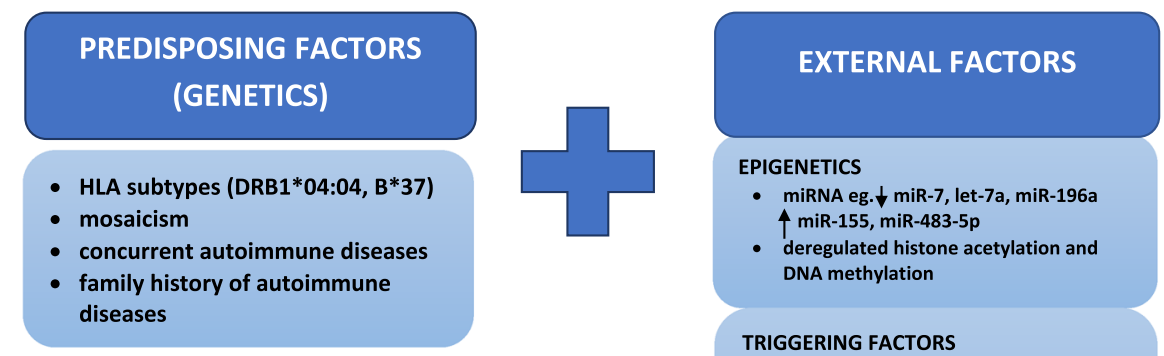

TRIGGERING FACTORS

- trauma/ surgery

- repeated friction

- injection/vaccination

- insects bites

- radiotherapy

- drugs

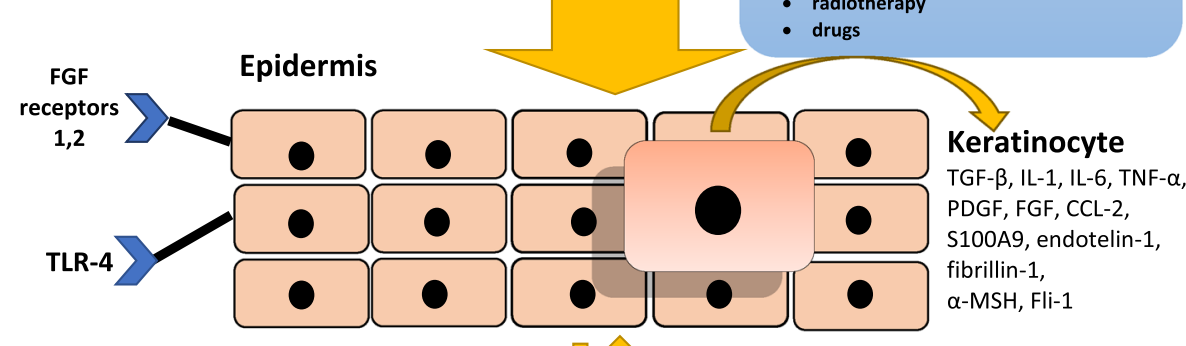

Dermis

Epidermal-dermal signalling pathways (Wnt, Jagged-Notch, Shh)

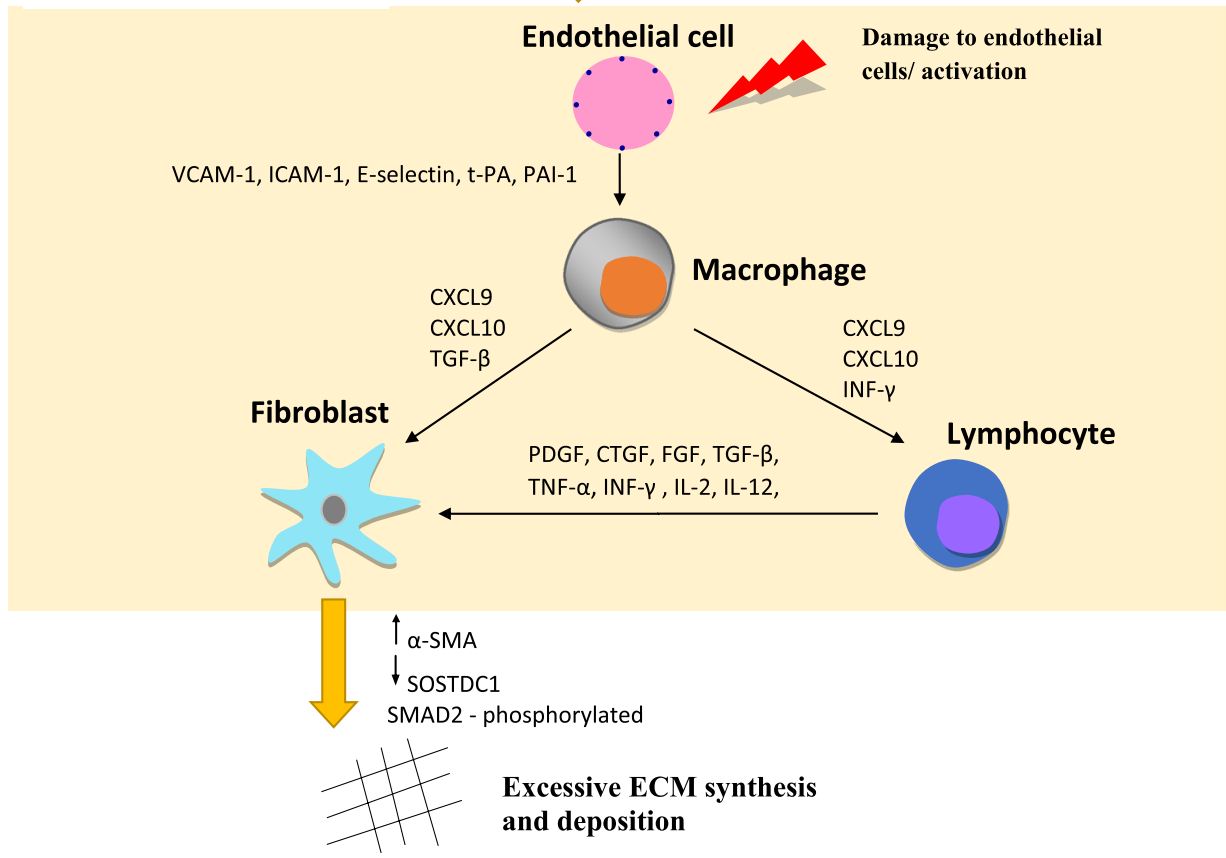

A number of external factors promote diseases onset in susceptible patients. Injuries, repeated friction (especially along with bra straps, waistline, groins), injections/vaccinations (hepatitis B, tetanus, vitamin B12), insect bites may induce localized scleroderma in about $16 \%$ of adults and 9\% of children [26]. Postirradiation (notably radiotherapy of breast cancer) and drug-induced (ex. bisoprolol, bleomycin, D-penicillamine, anti-TNF- $\alpha$ ) localized scleroderma have also been reported [26].

Triggering event in a susceptible person leads to activation of keretinocytes and production of many factors driving fibrosis such as transforming growth factor $\beta$ (TGF- $\beta$ ), tumor necrosis factor- $\alpha$ (TNF- $\alpha$ ), plateletderived growth factor (PDGF), fibroblast growth factor (FGF), friend leukaemia integrated transcription factor (Fli-1), endotelin-1 (ET-1), fibrillin-1, $\alpha$-melanocytestimulating hormone $(\alpha-\mathrm{MSH})$, interleukin 1 (IL-1), IL-6, S100A9 [26]. Production of protein S100A9 occurs further to epidermal stress and via toll-like receptor 4 (TLR4 ) induce fibroblast proliferation by potentiating TGF- $\beta$ activity [26]. Additionally, knocking down FGF receptors 1 and 2 results in activation of keratinocytes and release 
of profibrotic factors (S100A9, IL-1 [26]). Keratinocytes interact with endothelial cells and fibroblasts through signalling pathways including Wnt, Sonic hedgehog (Shh) and Jagged-Notch [26].

Damage to endothelial cells due to infection, inflammation, autoimmune reaction or skin injury leads to the secretion of cytokines and adhesion molecules (intracellular adhesion molecule-1 (ICAM-1), vascular cell adhesion molecule-1 (VCAM-1), E-selectin, CXCL-8), resulting in the recruitment of immune cells (lymphocytes, macrophages) $[3,27,29]$. These immune cells produce principal fibrotic growth factors such as transforming growth factor beta (TGF- $\beta$ ), connective tissue growth factor (CTGF) and platelet-derived growth factor (PDGF), driving fibroblast activation, proliferation and differentiation $[3,27,31]$. Badshah and co-workers have recently reported that fibroblasts exhibit continuous activation of phosphorylated SMAD2 which is important in TGF- $\beta$ signalling pathway [4]. Moreover, they shed light on upregulation of $\alpha$-SMA ( $\alpha$-smooth muscle actin) and downregulation of SOSTDC1 (sclerostin domain containing 1 -antagonist of TGF- $\beta$ and Wnt signalling pathways) in these cells [4]. Both persistent fibroblast proliferation and differentiation into myofibroblasts results in excessive extracellular matrix (ECM) synthesis and deposition [3, 27]. Myofibroblasts arise from a variety of sources including circulating fibroblasts, resident mesenchymal cells, epithelial and endothelial cells in processes defined as epithelial-to-mesenchymal transition (EMT) [3, 27].

\section{MicroRNA}

MicroRNAs belong to small, noncoding RNAs that represent crucial regulators of cell differentiation, proliferation, apoptosis and immune response [25, 28]. Thus far, according to miRBase, 1917 microRNAs encoding sequences have been determined in the human genome. The biogenesis and maturation of microRNAs take place in several steps (Fig. 2). Synthesis of miRNA starts within nucleus where RNA polymerase II transcribes genes encoding miRNA to form primary microRNA (pri-microRNA) - a steam loop structure that is composed of several hundred to few thousands nucleotides $[13,28]$. The primary transcript is cleaved by microprocessor complex (RNase III enzyme Drosha and DGCR8) into premature miRNA (pre-microRNA) that consists of 60-70 nucleotides [5, 8]. Pre-microRNA is then translocated into the cytoplasm by exportin-5 (XPO5) and further processed by endonuclease Dicer into mature double-stranded microRNA (22 nucleotides long) [3, 28]. One strand (passenger stands/complementary stand) is destructed by Argonaute proteins, the other strand (guide strand/mature strand) associates with RISC (RNA-induced silencing complex). The mature miRNA interact with 3'untranslated region
(UTR) of target mRNA by one of two mechanisms of gene regulation-mRNA degradation or repression of mRNA translation, depending on the degree of complementarity of microRNA and mRNA [13, 28]. Compelling complementarity lead to mRNA degradation, whereas confined complementarity guide repression of mRNA translation $[3,28]$.

MicroRNAs are now considered to play a pivotal role in the regulation of certain processes related to the development of number human diseases such as cancers, cardiovascular, autoimmunologic, neurodegenerative, liver and skin diseases [1, 23]. Among dermatologic ones, psoriasis, atopic dermatitis, allergic contact dermatitis, lichen planus, localized and systemic scleroderma, bullous diseases, alopecia, vitiligo and skin cancers have been established $[1,23]$.

\section{MicroRNA and fibrosis}

Recent reports have shown that several microRNAs participate in regulation of processes that drive fibrosis, including transforming growth factor (TGF- $\beta$ ) signalling, fibroblast proliferation and differentiation, extracellular matrix proteins deposition and epithelial-to-mesenchymal transition (EMT) [3, 18, 34]. Upregulation of some profibrotic microRNAs results in fibrosis, whereas antifibrotic miRNAs suppress this process and may be knock-down in fibrosis [13].

According to literature, MiRNAs involved in TGF- $\beta$ signalling cascade include miRNA-18, miRNA-20, miRNA21, miRNA-23b, miRNA-29, miRNA-140-5p, miRNA146a, miRNA-206 [3, 16]. MiRNA supposed to regulate fibroblast proliferation and differentiation are miRNA-21, miRNA-31, miRNA-146a and miRNA-200 family [3, 16]. Molecules affecting extracellular matrix synthesis and deposition are miRNA-let-7a, miRNA-7, miRNA-26a, miRNA29, miRNA-129-5p, miRNA-133a, miRNA-133b, miRNA150, miRNA-196a [3].

\section{Materials and methods}

A review of the literature published from January 2000 to October 2018 was performed using MEDLINE, Scopus, Web of Science, Clinical Key databases according to PRISMA (Preferred Reporting Items of Systematic Reviews and Meta-Analyses) guidelines. A search of databases was conducted utilizing MeSH terms: "localized scleroderma" OR "morphea" OR "circumscribed scleroderma" OR "dermatosclerosis" AND "microRNA" OR "micro RNA" OR "miRNA" OR "miRNAs". 
Fig. 2 The biogenesis and maturation of microRNAs [3, 26]

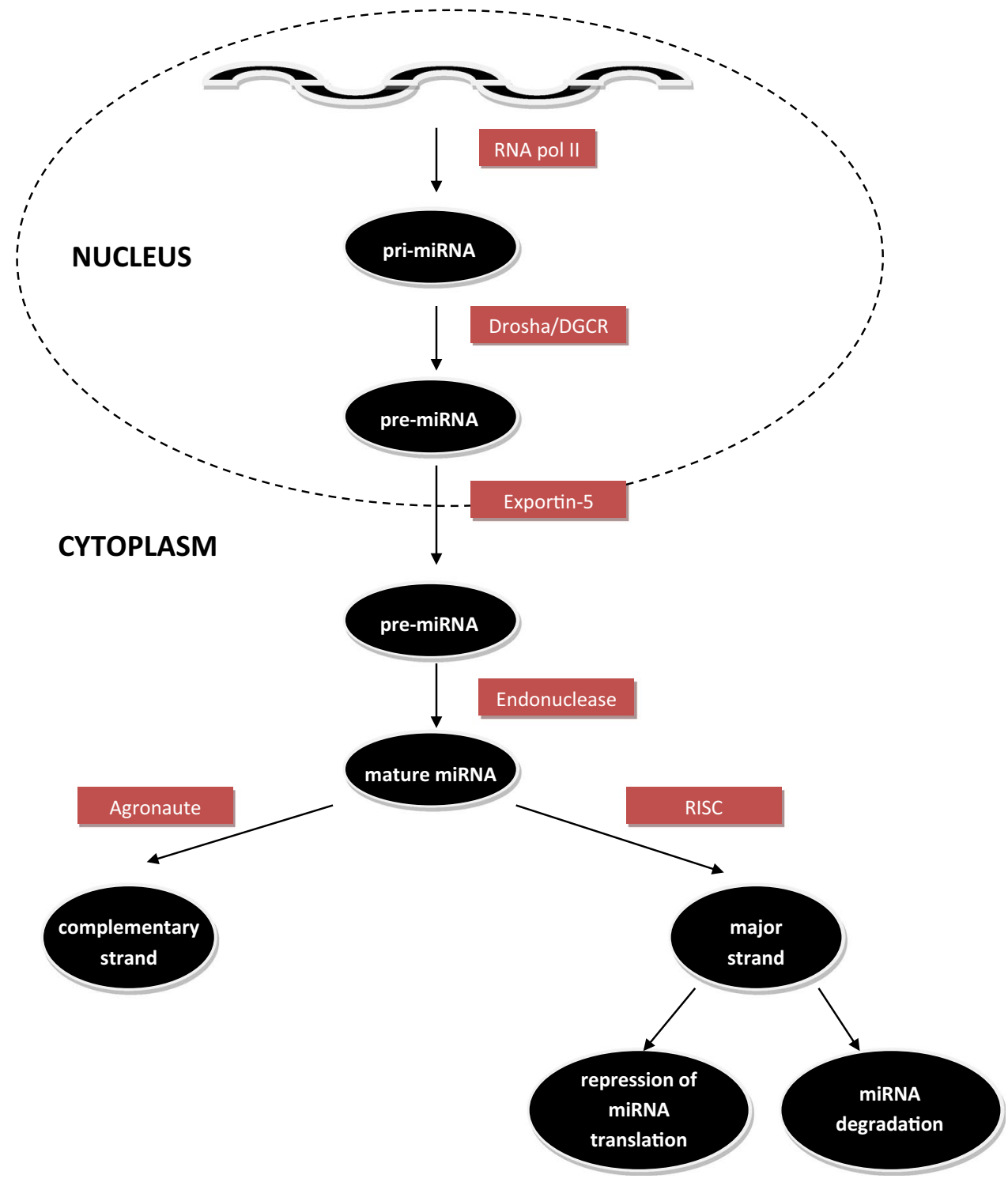

\section{Results}

The preliminary investigation revealed 132 articles (Fig. 3). After duplicates were removed, a total of 9 results were screened. Of the remaining records, 1 was irrelevant to localized scleroderma and 3 were review articles on systemic scleroderma. This resulted in 5 clinical studies that met inclusion criteria and were included in this review (Table 1).

\section{MicroRNA-7}

The role of miRNA-7 in the function of the skin remains unexplained, however, modified miR-7 expression has been stated in wound healing process and fibrotic skin diseases (scleroderma, dermatomyositis) [11]. Etoh and co-workers reported downregulated levels of miRNA-7 in 30 serum samples of localized scleroderma patients as well as skin biopsies of 5 patients with LoSc and 3 patients with keloid [9]. Among localized scleroderma patients, mean miR-7 levels were lower in individuals with linear type of LoSc, comparing those with morphea or generalized LoSc [9]. Serum microRNA-7 concentration was inversely correlated with disease duration and mLoSSI (Localized Scleroderma Skin Activity Index), but not significant [9]. Knocking down of microRNA-7 in normal fibroblasts caused overexpression of COL1A2 which was demonstrated to be a direct target of miR-7 [11, 25]. Therefore, miR-7 may carry in pathogenesis of localized scleroderma due to overexpression of type II collagen. Serum miR-7 can be considered as a promising biomarker and modulation of miR-7 in dermal fibroblast may be favourable method of treatment of LoSc. [8, 21]. 
Fig. 3 Literature searched based on PRISMA protocol
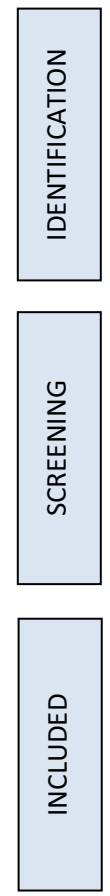

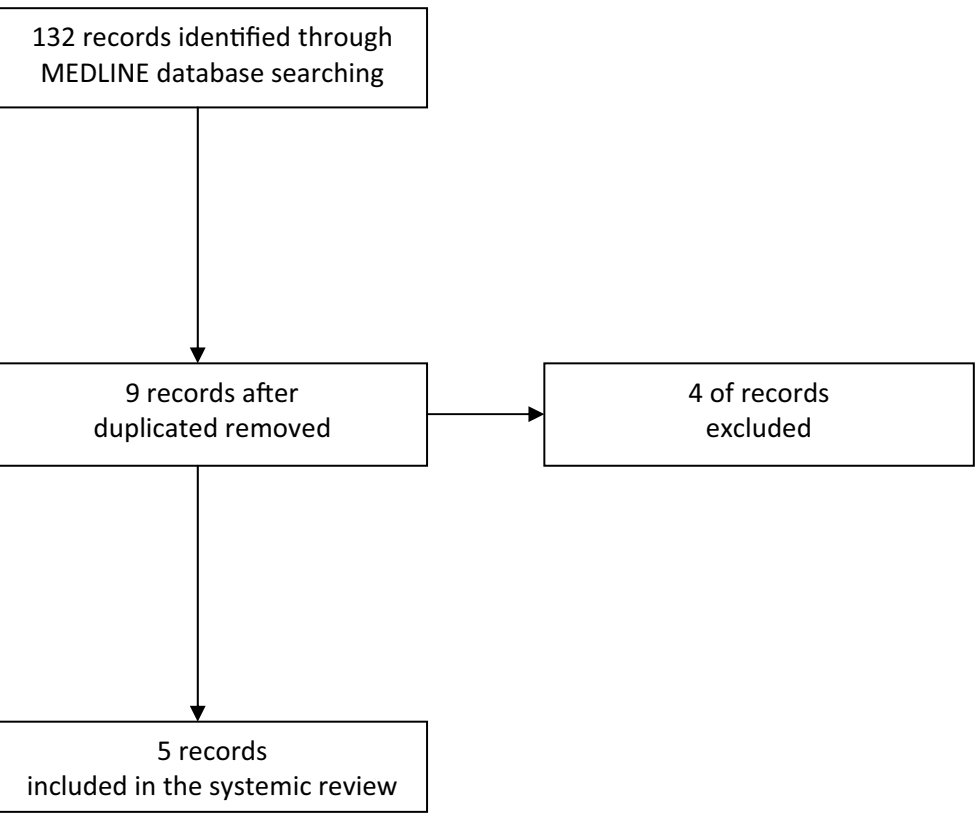

Table 1 Studies included in this systemic review [6, 8, 16, 17, 29]

\begin{tabular}{|c|c|c|c|c|c|}
\hline Study & Etoh et al. 2013 [8] & Makino et al. 2013 [16] & Makino et al. 2014 [17] & Yan et al. 2016 [29] & Chouri et al. 2018 [6] \\
\hline $\begin{array}{l}\text { Study group (LoSc } \\
\text { patients) }\end{array}$ & $30 / 5$ & $32 / 7$ & $34 / 3$ & 7 & 22 \\
\hline $\begin{array}{l}\text { No of patients with circum- } \\
\text { scribed LoSc }\end{array}$ & 15 & 19 & No data & No data & No data \\
\hline $\begin{array}{l}\text { No of patients with liner } \\
\text { LoSc }\end{array}$ & 10 & 8 & No data & No data & No data \\
\hline $\begin{array}{l}\text { No of patients with gener- } \\
\text { alized LoSc }\end{array}$ & 5 & 5 & No data & No data & No data \\
\hline Control group & $20 / 7$ & $17 / 7$ & $22 / 3$ & 9 & 9 \\
\hline Material & Serum/biopsy & Serum/biopsy & Serum/biopsy & Biopsy & Serum \\
\hline miRNA & miRNA-7 & miRNA-let7a & miRNA-196a & miRNA-155 & miRNA-483-5p \\
\hline Regulation & Downregulation & Downregulation & Downregulation & Upregulation & Upregulation \\
\hline
\end{tabular}

\section{miRNA-let 7a}

The let-7 family miRNAs were the first discovered human microRNAs [12, 24]. MicroRNA-let 7a is another molecule that is knockdowned in both serum and dermal fibroblast of LoSc patients. Makino and collaborators demonstrated that miR-let 7a levels in 7 localized scleroderma skin biopsies and 7 systemic scleroderma (SSc) skin samples were significantly decreased, comparing with 7 healthy skin biopsies and 5 keloid skin samples [17]. The decline of miRNA-let 7 a concentration in LoSc skin samples was greater that in systemic scleroderma ones [17]. Serum miRNA-let 7a levels in patients with localized scleroderma were remarkably lower than those in the control cohort which is in accordance with the reduction of miRNA let-7a in LoSc skin [17].
Furthermore, there was no prominent difference in the levels of miRNA-let $7 \mathrm{a}$ among the three groups of localized scleroderma (19 morphea, 8 linear, 5 generalized) [17]. Authors reported no correlation between the serum level of microRNA-let $7 \mathrm{a}$ and following parameters: number of lesions, disease duration, soluable IL-2R and ss-DNA [17]. Effectiveness of miRNA-let 7a mimics was successfully verified in a BALB/cAJcl mouse model of bleomycininduced skin fibrosis [17]. Let 7a oligonucleotides were injected transdermally into the shaved back of mice which caused increase in let-7a concentration in the skin and linked improvement in skin fibrosis $[3,17]$. Authors attempted to elucidate the mechanism that mediates reduced expression of microRNA-let 7a in scleroderma fibroblasts [17]. They 
stimulated healthy fibroblasts with exogenous TGF- $\beta 1$ which guided reduced microRNA-let 7a expression [17].

\section{miRNA-196a}

MicroRNA-196a is a putative regulator of $\alpha 1$ (I) and $\alpha 2$ (I) chains that are components of type I collagen. Makino and co-workers reported that depletion of miRNA-196a level in 3 localized scleroderma skin biopsies was statistically significant compared with 3 keloid skin samples and 3 normal skin biopsies [18]. Consistent with knockdown of microRNA196a in LoSc skin, the serum concentrations of miRNA196a were remarkably diminished in patients with localized scleroderma (34) in contrast to healthy controls (22) [18]. Nonetheless, there was no correlation between serum levels of miRNA-196a and types of LoSc, clinical manifestations or laboratory tests. In cultured dermal fibroblasts, knockdown of miRNA-196 was normalized by depletion of TGF- $\beta$ level [18, 25]. Inhibition of microRNA-196a enhanced type I collagen synthesis in normal fibroblast, while transfection of the microRNA-196 mimic lead to downregulation of collagen in scleroderma fibroblast $[11,18,25]$. Two years earlier, the same authors reported that in systemic scleroderma there was no prominent variation between healthy controls and patient group [10]. Divergences between systemic and localized scleroderma may be caused by different extension of skin fibrosis [18]. LoSc lesions tend to be more severe, affecting deeper structures lying beneath the skin (subcutaneous tissue, fascia, muscles, bones) in contrast to systemic scleroderma lesions [18].

\section{miRNA-155}

MicroRNA-155 is a profibrotic molecule that has been identified to regulate endothelial-to-mesenchymal transition (EndoMT) - differentiation by which endothelial cells (ECs) lose their specific structure/junctions and acquire myofibroblast-like features [6, 19]. Moreover, miRNA-155 is a pivotal, negative regulator of the inflammatory response of the pattern recognition receptors (toll-like receptors, TLR) [22]. One of the foremost ligands for TRL4 is lipopolysaccharide (LPS) which has been reported to alter the expression level of miR-155 [22]. Yan and co-workers demonstrated that miRNA-155 expression was upregulated in scleroderma patients' skin (19) as opposed to control group (9) [33]. Furthermore, the expression of miRNA-155 was higher in localized scleroderma samples (9) comparing with systemic scleroderma ones (12) [33]. Authors showed that miRNA-155 expression in the lesional skin correlated positively with the expansion of skin involvement in SSc patients [33]. Yan and collaborators found that miRNA-155 regulated Akt and Wnt/ $\beta$-cathenin pathways [2,33]. MiRNA-155 mimics firmly diminished the degradation of $\beta$-cathenin and increased the phosphorylation of Akt, whereas miRNA-155 inhibitor acted inversely to above pathways $[2,33]$. The study revealed an intending, innovating treatment approach to target miRNA155 [33]. Two weeks after bleomycin-induced skin fibrosis in mice models, topical anagomiR-155 was applied, revealing significant depletion of dermal thickening and collagen desposition [33]. These promising results imply that epicutaneous antagonist of miRNA-155 could be favourable to patients with limited subtypes of localized scleroderma [33]. Nevertheless, onwards studies on human skin samples are essential [33].

\section{miRNA-483-5p}

MicroRNA-483-5p is a molecule supposed to be a specific marker for skin fibrosis. Chouri and co-workers demonstrated that miRNA-483-5p was upregulated both in localized scleroderma (22) and systemic scleroderma (107) serum samples, unlike other autoimmunologic diseases [systemic lupus erythematosus (33), Sjögren's syndrome (23)] [7]. These results indicate that miRNA-483-5p may be distinctive for conditions characterized by fibrosis of the skin [7]. Furthermore, researchers exhibited that miRNA483-5p overexpression in endothelial cells increased the transcriptional levels of $\alpha$-SMA (alpha-smooth muscle actin) and SM22A (smooth muscle protein 22-alpha) —indicators of myofibroblast differentiation [7]. Additionally, miRNA483-5p decreased the level of Fli-1 (friend leukemia virus integration 1) - a negative regulator of extracellular matrix [7]. In previous studies, miRNA-483-5p was revealed to target Matn3 (matrillin 3) and TIMP2 (tissue inhibitor of metalloproteinase 2) to enhance extracellular matrix degradation, chondrocyte hypertrophy and cartilage angiogenesis [32].

\section{Summary}

In this review, we summarized 5 clinical studies on microRNAs in localized scleroderma. The expression of miRNA-7, let 7a, 196a, 155, 483-5p was up- or downregulated, depending on their properties. Although scientists could not find any significant correlations between serum/skin microRNAs levels and clinical/laboratory findings, this may be due to small patient cohort and rarity of the disease. The delay in diagnosis and treatment of localized scleroderma may lead to uncontrolled progression of the disease and irreversible complications [18]. Therefore, miRNAs levels may be useful biomarkers of skin sclerosis severity that reflects collagen overexpression and facilitate progress towards appropriately assessing the disease. 


\section{Compliance with ethical standards}

Conflict of interest The authors declare that they have no conflict of interest.

Ethical approval This article does not contain any studies with human participants or animals performed by any of the authors.

Open Access This article is distributed under the terms of the Creative Commons Attribution 4.0 International License (http://creativeco mmons.org/licenses/by/4.0/), which permits unrestricted use, distribution, and reproduction in any medium, provided you give appropriate credit to the original author(s) and the source, provide a link to the Creative Commons license, and indicate if changes were made.

\section{References}

1. Ardekani AM, Naeini MM (2010) The role of microRNAs in human diseases. Avicenna J Med Biotechnol 2:161-179

2. Artlett CM, Sassi-Gaha S, Hope JL et al (2017) Mir-155 is overexpressed in systemic sclerosis fibroblasts and is required for NLRP3 inflammasome-mediated collagen synthesis during fibrosis. Arthritis Res Ther 19:144. https://doi.org/10.1186/s1307 5-017-1331-z

3. Babalola O, Mamalis A, Lev-Tov H, Jagdeo J (2013) The role of microRNAs in skin fibrosis. Arch Dermatol Res 305:763-776. https://doi.org/10.1007/s00403-013-1410-1

4. Badshah II, Brwon S, Weibel L (2019) Differential expression of secreted factors SOSTDC1 and ADAMTS8 cause profibrotic changes in linear morphea fibroblasts. Br J Dermatol 180:1135-1149

5. Banerjee J, Chan YC, Sen CK (2011) MicroRNAs in skin and wound healing. Physiol Genomics 43:543-556. https://doi. org/10.1152/physiolgenomics.00157.2010

6. Bijkerk R, de Bruin RG, van Solingen C et al (2012) MicroRNA-155 functions as a negative regulator of RhoA signaling in TGF- $\beta$-induced endothelial to mesenchymal transition. MicroRNA (Shariqah, United Arab Emirates) 1:2-10

7. Chouri E, Servaas NH, Bekker CPJ et al (2018) Serum microRNA screening and functional studies reveal miR-483-5p as a potential driver of fibrosis in systemic sclerosis. J Autoimmun 89:162-170. https://doi.org/10.1016/j.jaut.2017.12.015

8. Deng X, Su Y, Wu H et al (2015) The role of microRNAs in autoimmune diseases with skin involvement. Scand J Immunol 81:153-165. https://doi.org/10.1111/sji.12261

9. Etoh M, Jinnin M, Makino K et al (2013) microRNA-7 down-regulation mediates excessive collagen expression in localized scleroderma. Arch Dermatol Res 305:9-15. https://doi.org/10.1007/ s00403-012-1287-4

10. Honda N, Jinnin M, Kajihara I et al (2012) TGF-mediated downregulation of MicroRNA-196a contributes to the constitutive upregulated type I collagen expression in scleroderma dermal fibroblasts. J Immunol 188:3323-3331. https://doi.org/10.4049/ jimmunol.1100876

11. Horsham JL, Ganda C, Kalinowski FC et al (2015) MicroRNA-7: a miRNA with expanding roles in development and disease. Int J Biochem Cell Biol 69:215-224. https://doi.org/10.1016/j.bioce 1.2015.11.001

12. Izumiya Y, Jinnn M, Kimura $Y$ et al (2015) Expression of Let-7 family microRNAs in skin correlates negatively with severity of pulmonary hypertension in patients with systemic scleroderma. IJC Hear Vasc 8:98-102. https://doi.org/10.1016/J.IJCHA .2015 .06 .006
13. Jinnin M (2014) Various applications of microRNAs in skin diseases. J Dermatol Sci 74:3-8. https://doi.org/10.1016/j.jderm sci.2014.01.004

14. Knobler R, Moinzadeh P, Hunzelmann N et al (2017) European Dermatology Forum S1-guideline on the diagnosis and treatment of sclerosing diseases of the skin, part 1: localized scleroderma, systemic sclerosis and overlap syndromes. J Eur Acad Dermatol Venereol 31:1401-1424. https://doi.org/10.1111/jdv.14458

15. Kreuter A, Krieg T, Worm M et al (2016) German guidelines for the diagnosis and therapy of localized scleroderma. JDDG J der Dtsch Dermatologischen Gesellschaft 14:199-216. https://doi. org/10.1111/ddg.12724

16. Li Y, Zhang J, Lei Y et al (2017) MicroRNA-21 in skin fibrosis: potential for diagnosis and treatment. Mol Diagn Ther 21:633642. https://doi.org/10.1007/s40291-017-0294-8

17. Makino K, Jinnin M, Hirano A et al (2013) The downregulation of microRNA let-7a contributes to the excessive expression of type I collagen in systemic and localized scleroderma. J Immunol 190:3905-3915. https://doi.org/10.4049/jimmunol.1200822

18. Makino T, Jinnin M, Etoh M et al (2014) Down-regulation of microRNA-196a in the sera and involved skin of localized scleroderma patients. Eur J Dermatol 24:470-476. https://doi. org/10.1684/ejd.2014.2384

19. Manetti M, Romano E, Rosa I et al (2017) Endothelial-to-mesenchymal transition contributes to endothelial dysfunction and dermal fibrosis in systemic sclerosis. Ann Rheum Dis 76:924-934. https://doi.org/10.1136/annrheumdis-2016-210229

20. Mertens J, Seyger MMB, Thurlings RM et al (2017) Morphea and eosinophilic fasciitis: an update. Am J Clin Dermatol 18:491-512. https://doi.org/10.1007/s40257-017-0269-x

21. Miao C, Xiong Y, Yu H et al (2015) Critical roles of microRNAs in the pathogenesis of systemic sclerosis: new advances, challenges and potential directions. Int Immunopharmacol 28:626633. https://doi.org/10.1016/j.intimp.2015.07.042

22. O'Reilly S (2016) MicroRNAs in fibrosis: opportunities and challenges. Arthritis Res Ther 18:11. https://doi.org/10.1186/s1307 5-016-0929-x

23. Paul P, Chakraborty A, Sarkar D et al (2018) Interplay between miRNAs and human diseases. J Cell Physiol 233:2007-2018. https://doi.org/10.1002/jcp. 25854

24. Roush S, Slack FJ (2008) The let-7 family of microRNAs. Trends Cell Biol 18:505-516. https://doi.org/10.1016/j.tcb.2008.07.007

25. Ruksha TG, Komina AV, Palkina NV (2017) MicroRNA in skin diseases. Eur J Dermatol 27:343-352. https://doi.org/10.1684/ ejd.2017.3024

26. Saracino AM, Denton CP, Orteu CH (2017) The molecular pathogenesis of morphoea: from genetics to future treatment targets. $\mathrm{Br}$ J Dermatol 1:34-36. https://doi.org/10.1111/bjd.15001

27. Shroff A, Mamalis A, Jagdeo J (2014) Oxidative stress and skin fibrosis. Curr Pathobiol Rep 2:257-267. https://doi.org/10.1007/ s40139-014-0062-y

28. Singhvi G, Manchanda P, Krishna Rapalli V et al (2018) MicroRNAs as biological regulators in skin disorders. Biomed Pharmacother 108:996-1004. https://doi.org/10.1016/j.biopha.2018.09.090

29. Takehara K, Sato S (2005) Localized scleroderma is an autoimmune disorder. Rheumatol 44:274-279. https://doi.org/10.1093/ rheumatology/keh487

30. Torok KS, Li SC, Jacobe HM et al (2019) Immunopathogenesis of pediatric localized scleroderma. Front Immunol 10:1-11. https ://doi.org/10.3389/fimmu.2019.00908

31. Vettori S, Gay S, Distler O (2012) Role of microRNAs in fibrosis. Open Rheumatol J 6:130-139. https://doi.org/10.2174/18743 12901206010130

32. Wang H, Zhang H, Sun Q et al (2017) Intra-articular delivery of antago-miR-483-5p inhibits osteoarthritis by modulating matrilin 
3 and tissue inhibitor of metalloproteinase 2. Mol Ther 25:715727. https://doi.org/10.1016/j.ymthe.2016.12.020

33. Yan Q, Chen J, Li W et al (2016) Targeting miR-155 to treat experimental scleroderma. Sci Rep 6:20314. https://doi.org/10.1038/ srep20314

34. Yang C, Zheng S-D, Wu H-J, Chen S-J (2016) Regulatory mechanisms of the molecular pathways in fibrosis induced by microRNAs. Chin Med J (Engl) 129:2365. https://doi.org/10.4103/03666999.190677
Publisher's Note Springer Nature remains neutral with regard to jurisdictional claims in published maps and institutional affiliations. 